\title{
Modifications of the dielectric and thermal properties of vulcanized natural rubber due to iodine doping
}

\author{
M. D. da Silva and L. C. M. Miranda \\ Laboratório Associado de Sensores e Materiais, Instituto Nacional de Pesquisas Espaciais, Caixa Postal 515, \\ 12201-970 São José dos Campos, São Paulo, Brazil \\ (Received 8 November 1993)
}

\begin{abstract}
The marked changes in the dielectric and thermal properties of vulcanized cis-1,4-polyisoprene due to different iodine doping levels are investigated. The results show strong evidences of formation of conjugated sequences of unsaturated double bonds in the vulcanized polyisoprene backbone, through visible and infrared spectroscopic data. The dependence of the electrical conductivity, specific heat, and of the Young's modulus, upon doping, indicate the existence of a critical doping concentration for the onset of an order-disorder transition.
\end{abstract}

\section{INTRODUCTION}

Two decades have elapsed since the first demonstration $^{1}$ of the development of an electrically conducting polymer. Most of the research on conductive polymers has focused on conjugated polymers like polyacetylene, which have long series of alternating single and double bonds. The high degree of electronic density delocalization in these polymers lowers the energy gap between valence and conduction bands so that, upon doping with donor or acceptor species, the material exhibits a drastic change in electrical conductivity, ${ }^{2}$ sometimes comparable to that of metals even at room temperature. ${ }^{3}$ The current and potential applications of conductive polymers span a wide range of areas, going from simple flexible rubbery conductors to solar and electrochemical cells. The possibilities seem to be unlimited due to the ease with which polymers can be tailored and conformed.

From the specific point of view, a great debate arose four years ago regarding the basic conduction mechanism in these materials when Thakur and Elman ${ }^{4,5}$ made the discovery that nonconjugated polymers could also be made conductive by doping with small amounts of iodine. Thakur found that when natural rubber (cis-1,4polyisoprene), a nonconjugated polymer with isolated double bonds, is treated with $I_{2}$, it becomes ten orders of magnitude more conducting than native rubber, although still much less conducting than iodine-doped polyacetylene. This effect was also seen with gutta-percha (trans-1,4-polyisoprene) and with cis-1,4-poly (2,3dimethylbutadine), but not with cis-1,4,-polybutadiene. The difference in conductivity among these materials was attributed by this author to the presence of methyl groups at double-bond positions in the first three polymers and their absence in cis-1,4-polybutadiene. When the first three polymers are oxidized with $\mathrm{I}_{2}$, an electron is removed from the $\pi$-bonding orbital of each double bond, resulting in the formation of an ethylenic radical cation. The methyl groups facilitate this reaction by contributing sufficient electron density to the double bonds to make them susceptible to oxidation by $I_{2}$. This radical cation model says that conduction takes place by charges moving along the charged sites of the polymer. Thakur's finding was later confirmed by Shang, Pramanick, and Hudson $^{6}$ who have also extended it to additional nonconjugated polymers. These authors, ${ }^{6}$ however, showed that methyl groups at the double-bond position are not required for conductivity and suggested that conduction is likely to occur by electronic transport through polyiodine chains. More recently, Dai and White ${ }^{7}$ have demonstrated that $I_{2}$ doping of 1,4-polyisoprene produces, by double-bond shift reactions, ${ }^{8,9}$ conjugated sequences of unsaturated double bonds in the polyisoprene backbone. The formation of conjugated conducting polymer from $\mathbf{I}_{2}$ doping of 1,4-polyisoprene was investigated by these authors both in solution and in cast films. The results of Dai and White indicated that the previously ${ }^{4-6}$ suggested mechanism for the chemical transformations taking place during the $\mathrm{I}_{2}$ doping of polyisoprene should be treated with some caution.

In this paper, we present a detailed monitoring of the changes in the electrical and thermal properties of vulcanized cis-1,4-polyisoprene as a function of the iodinedoping concentration. Both the initial polymer matrix as well as the iodine process adopted differed considerably from those used in the previous works. ${ }^{4-7}$ The onset of the changes induced by the $I_{2}$ doping on the above physical properties was further supported by additional data on the kinetics of the doping process as manifested by the changes in the visible and infrared spectra and in the tensile strength measurements of the doped samples as a function of the iodine concentration.

\section{EXPERIMENT}

The vulcanized natural rubber samples were obtained from Johnson and Johnson Co. in the form of films of about $70 \mu \mathrm{m}$ thickness. Reagent grade chemicals were used without further purification. The iodine doping was carried out by immersion in iodine-methanol solutions. The samples were cut in the shape of disks of $25 \mathrm{~mm}$ diam. After being thoroughly washed in methanol they 
were fixed between two, tambourinelike, PVC rings, and immersed in previously prepared iodine-methanol solutions of different $I_{2}$ concentrations for $15 \mathrm{~h}$. The removal from the doping solutions was followed by a thorough rising in methanol again to remove any iodine excess particles left on the sample surface. The concentrations of the iodine-methanol doping solutions used, referred from now on as doping concentrations, ranged between 2 and $24 \mathrm{~g} / \mathrm{l}$. The iodine doping using the more concentrated doping solution, resulted in a black, rigid, and somewhat corrugated sample, which tends to make it more difficult to assembling them in the dielectric and thermal properties measuring setups.

The optical-absorption data between 250 and $900 \mathrm{~nm}$ was obtained using a Lambda 15 VIS-UV Perkin-Elmer spectrometer, whereas for the infrared spectra between 4400 and $450 \mathrm{~cm}^{-1}$ we have used a 1600 Fourier transform infrared (FTIR) Perkin-Elmer spectrometer. The dielectric measurements were carried out using a Hewlett-Packard model 4274A LCR meter operating at 1 $\mathrm{kHz}$. The thermal properties measurements were accomplished using two different homemade photoacoustic setups for measuring the thermal diffusivity and the thermal effusivity, as discussed in Refs. 10 and 11. The thermal diffusivity was measured in a heat-transmission photoacoustic cell configuration, whereas the thermal effusivity was measured in a conventional frontal heat deposition photoacoustic configuration. Finally, the tensile testing was carried out on an INSTRON model 1131 tensile testing machine. The error bars used in the presentation of the experimental data in this paper, correspond to a series of, at least, five experimental runs.

\section{VISIBLE AND INFRARED SPECTRA}

Our polyisoprene samples exhibited an appreciable color change upon iodine treatment, similarly to the previous works in polyisoprene $e^{4,5}$ and polybutadiene. ${ }^{6}$ They changed color from a colorless state for the undoped sample to a metallic black luster for the heavily doped samples, indicating that the polymer reacted with iodine. This is evident in the optical-absorption spectra of samples treated with different doping solutions, as shown in Figs. 1 and 2. Traces $c$ and $d$ in Fig. 1 refer to the absorption spectra of freshly doped samples with doping solution concentrations of 4 and $8 \mathrm{~g} / \mathrm{l}$, respectively, whereas traces $b$ and $e$ are the corresponding spectra of these samples after a seven-day aging; trace $a$ is the absorption spectrum for the undoped cis-1,4,-polyisoprene. Figure 2 shows the absorption spectra evolution for seven-day aged samples, as a function of the doping concentration.

The first point to be noted in both figures is that the absorption edge shifts towards longer wavelengths as the iodine-doping concentration increases. This is in agreement with the visually observed sample darkening. The second interesting aspect is that the effect of the sample aging seems to be to induce two distinct kinetics of the absorption spectra as a function of the doping concentration. For a doping concentration of $8 \mathrm{~g} / 1$ (traces $d$ and $e$ in Fig. 1), the aging effect is such that the absorption

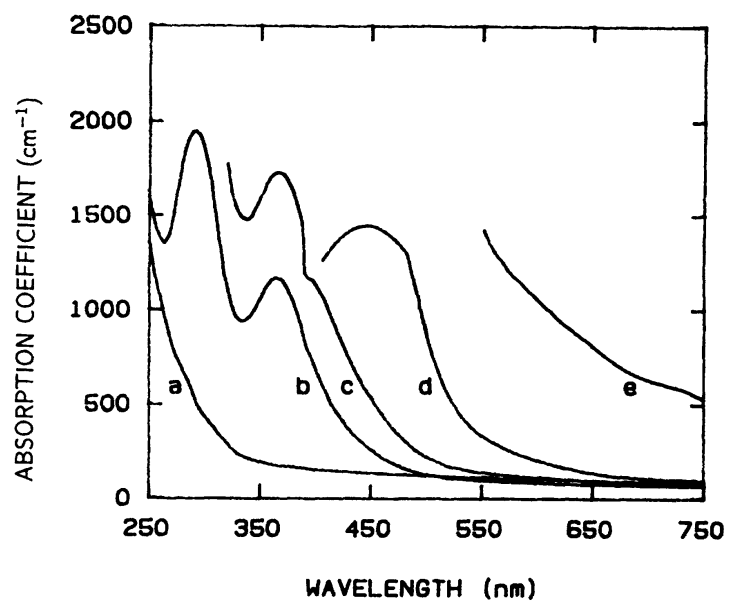

FIG. 1. Optical-absorption spectra of vulcanized cis-1,4polyisoprene for an undoped sample $(a)$, for seven-day aged $(b)$, and freshly $(c)$ doped samples using a 4-g/1 solution concentration, and for seven-day aged $(e)$ and freshly $(d)$ doped samples using a $8-\mathrm{g} / 1$ solution concentration.

band increases in intensity and shifts towards long wavelengths, as the sample is left aging. In contrast, for a doping concentration of $4 \mathrm{~g} / 1$ (traces $b$ and $c$ in Fig. 1), the absorption band weakens and shifts towards smaller wavelengths, as we let the sample age. As suggested by Dai and White, ${ }^{7}$ this shift in the absorption edge to smaller wavelengths may reflect a decrease in the average length of the polyisoprene segments. In fact, the absorption spectra of freshly and seven-day aged samples, similar to those shown in Fig. 1, was measured for samples doped with solution concentrations ranging from 2 to 20 $\mathrm{g} / \mathrm{l}$. We have found that the doping concentration of 6 $\mathrm{g} / \mathrm{l}$ seems to act as a divider for the iodine incorporation into the polyisoprene. For doping levels greater than 6 $\mathrm{g} / \mathrm{l}$, the samples exhibits an intensified absorption spectra shifted towards long wavelengths, as a function of aging,

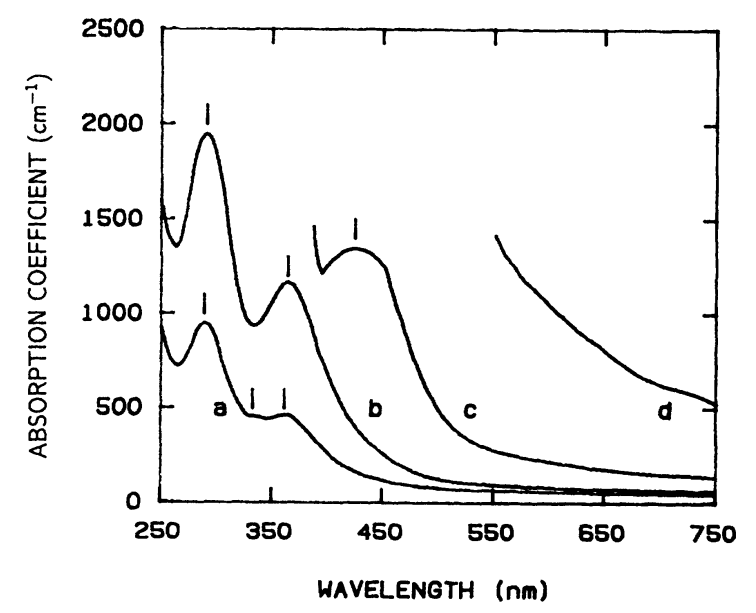

FIG. 2. Optical-absorption spectra of seven-day aged vulcanized cis-1,4-polyisoprene samples doped with iodine solution concentrations of $2(a), 4(b), 6(c)$, and $8(d) \mathrm{g} / 1$. The vertical lines in these plots refer to the absorption peak positions of conjugated sequences of double bonds. 
TABLE I. Absorption band positions of $\mathrm{H}-(\mathrm{CH}=\mathrm{CH})-\mathrm{H}$ sequences, taken from Refs. 7 and 12.

\begin{tabular}{cc}
\hline $\begin{array}{c}\text { Number of conjugated } \\
\text { double bonds, } n\end{array}$ & $\begin{array}{c}\text { Absorption band } \\
\text { peak wavelength } \\
\text { (nm) }\end{array}$ \\
\hline 3 & 268 \\
4 & 304 \\
5 & 334 \\
6 & 364 \\
7 & 390 \\
8 & 410 \\
9 & 428 \\
10 & 447 \\
\hline \hline
\end{tabular}

similarly to curves $d$ and $e$ of Fig. 1. For doping concentrations below $6 \mathrm{~g} / 1$, the aging effect manifests itself as producing a shift towards short wavelengths and a decrease in intensity. Furthermore, this short-wavelength shifting, for lightly doped samples, is accompanied by the appearance of several bands in the short-wavelength region (cf., curves $b$ and $c$ in Fig. 1), attributed ${ }^{7}$ to the formation of various conjugated sequences $(-\mathrm{CH}=\mathrm{CH}-$ ) in the polyisoprene chains. In Table I we list the wavelengths where the strongest absorption bands occur for the various $\mathrm{H}-(\mathrm{CH}=\mathrm{CH})_{n}-\mathrm{H}$ conjugated compounds. ${ }^{12}$ The good agreement between the absorption peak positions in Fig. 2 and those of Table I suggests that, indeed, conjugated sequences with up to 9 double bands have been introduced into the vulcanized cis-1,4polyisoprene chains, as in the case of trace $c$ of Fig. 2. The appearance of bands at $425 \mathrm{~nm}(n=9)$ (trace $c$ in Fig. 2) and at $446 \mathrm{~nm}(n=10)$ (trace $d$ in Fig. 1) at high doping levels suggests a continued growth in conjugation length with the iodine-doping concentration.

The FTIR spectra of an undoped and doped, sevendays aged, vulcanized cis-1,4-polyisoprene samples are shown in Fig. 3. Trace $a$ in Fig. 3 refers to the undoped sample whereas traces $b$ and $c$ refer to samples doped with solution concentration of 10 and $20 \mathrm{~g} / 1$, respective1y. The marked changes in the IR spectra are the almost complete disappearance of the isolated $\mathrm{C}=\mathrm{C}$ bending and stretching modes, ${ }^{4,5}$ as well as of the isolated $\mathrm{CH}$ wagging $\operatorname{mode}^{6}$ of the native cis-1,4-polyisoprene at 836 , 1663 , and $930 \mathrm{~cm}^{-1}$, respectively, upon heavy iodine doping.

The continuous decrease of the absorption coefficient of these bands, as a function of the doping solution concentration, is explicitly shown in Fig. 4. The solid lines in this figure represent the absorption coefficient data fitting to a logistic curve, namely,

$$
f(x)=f o+\Delta f \frac{\exp [(x-x o) / \Delta x]}{1+\exp [(x-x o) / \Delta x]} .
$$

It follows from Fig. 4 that up to a doping concentration of $6 \mathrm{~g} / 1$ the absorption coefficient decrease typically less than 20\%; above a 6-g/1 concentration, the lines exhibit a very rapid decrease upon iodine doping. These rapidly decreasing bands (corresponding to the $\mathrm{C}=\mathrm{C}$ vibrational modes) for doping concentrations above $6 \mathrm{~g} / 1$ is consistent with the observed loss of flexibility of our samples upon iodine doping. This enhancement of the sample rigidity at high doping levels may be attributed to interchain crosslinking, as observed for other conducting polymers.

The other interesting features of the FTIR spectra in Fig. 3 are the appearance of broad bands at 1545 and $1605 \mathrm{~cm}^{-1}$ and the evolution of the weak absorption bands at 1700 and $1740 \mathrm{~cm}^{-1}$, upon doping. The band at $1740^{-1}$ is attributed to carbonyl compounds naturally occurring in the material (natural rubber), which tends to disappear in photo-oxidation experiments. ${ }^{13}$ The 1700 $\mathrm{cm}^{-1}$ absorption band is also attributed to carbonyl compounds resulting from photo-oxidation, and it appears simultaneously with the disappearance of the $1740 \mathrm{~cm}^{-1}$ band. The evolution of the absorption coefficients of these two bands, as a function of the doping concentration, is shown in Fig. 5. Finally, in Fig. 6 we show the

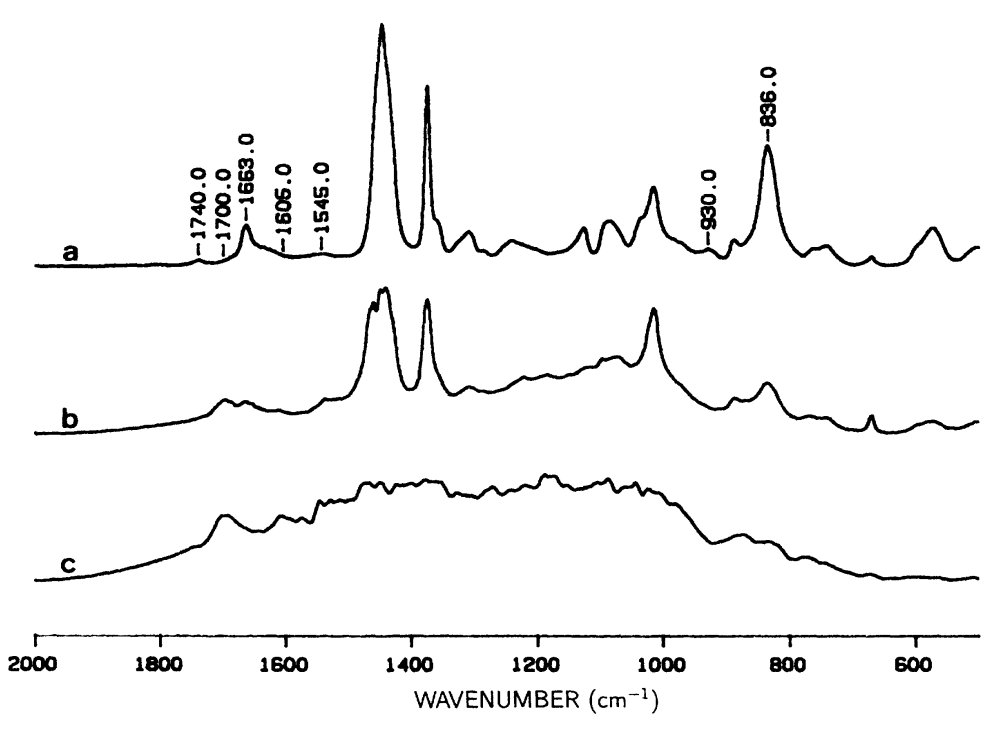

FIG. 3. FTIR spectra of seven-day aged vulcanized cis-1,4-polyisoprene samples. Trace $a$ refers to an undoped sample, whereas traces $b$ and $c$ refer to samples treated with 10and $20-\mathrm{g} / 1$ doping solution concentrations, respectively. 


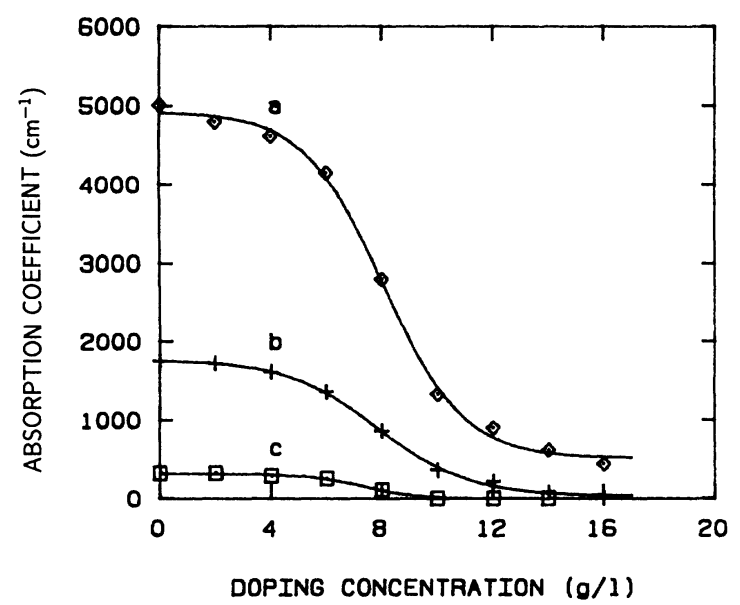

FIG. 4. Absorption coefficient of the $836 \mathrm{~cm}^{-1}(a), 1663$ $\mathrm{cm}^{-1}(b)$, and $930 \mathrm{~cm}^{-1}(c)$ bands of iodine doped vulcanized cis-1,4-polyisoprene samples, as a function of the doping solution concentration.

dependence of the absorption coefficients of the 1545 and $1605 \mathrm{~cm}^{-1}$ absorption bands, as a function of the doping concentration. These bands have been associated by Dai and White ${ }^{7}$ with the sequences of conjugated double bonds based upon the literature data ${ }^{14,15}$ of conjugated double-bond compounds. This figure tells us that in the concentration region where the isolated double-bond vibrational modes of the native chains decrease very rapidity (namely, concentrations greater than $6 \mathrm{~g} / 1$ ), the conjugated double-bond vibration modes exhibit a linear growth upon doping.

\section{DIELECTRIC PROPERTIES}

To make sure that our measurements reflected the optimum effect of the iodine doping we have checked for the aging effect on the dielectric measurements. The

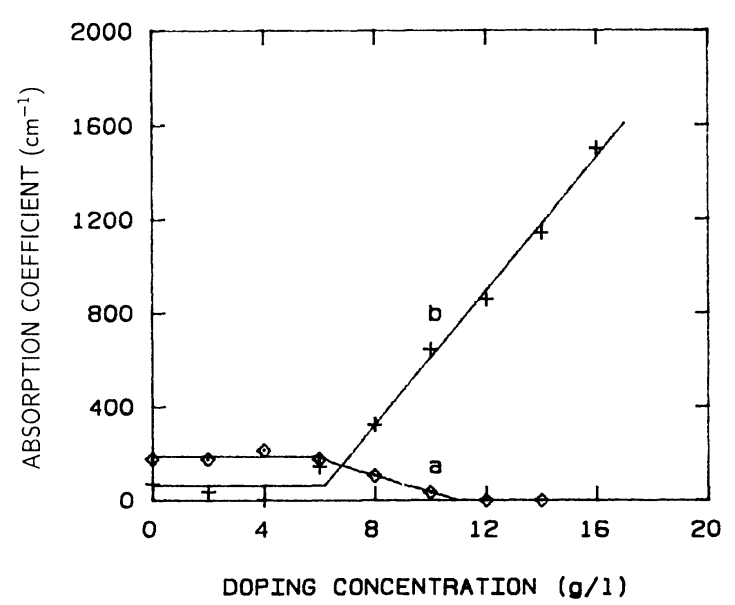

FIG. 5. Absorption coefficients of the $1740 \mathrm{~cm}^{-1}(a)$ and $1700 \mathrm{~cm}^{-1}(b)$ bands of iodine-doped vulcanized cis-1,4polyisoprene samples, as a function of the doping solution concentration.

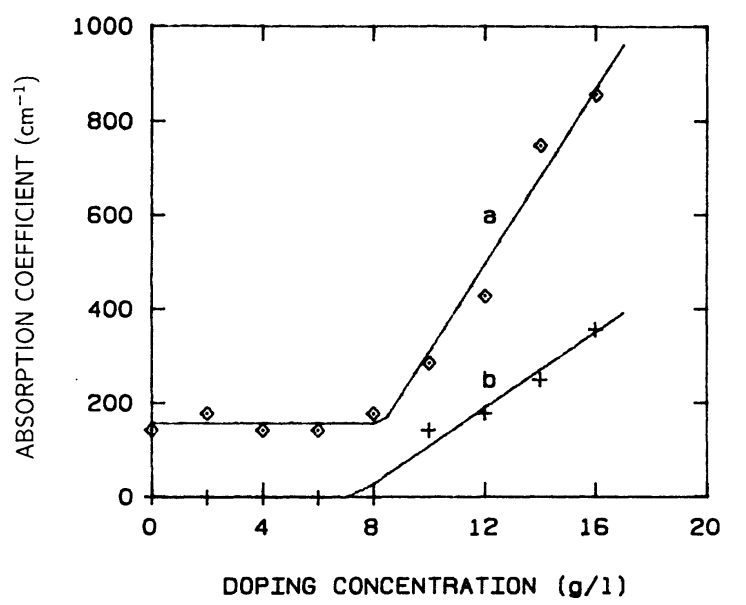

FIG. 6. Absorption coefficients of the $1545 \mathrm{~cm}^{-1}(a)$ and 1605 $\mathrm{cm}^{-1}(b)$ bands of iodine doped vulcanized cis-1,4-polyisoprene samples, as a function of the doping solution concentration.

reason for this is that, as discussed in the previous section, the optical-absorption spectra exhibited two distincts time behaviors depending upon the level of iodine doping.

In Fig. 7 we show the typical aging curves of the ac electrical conductivity, at $1 \mathbf{k H z}$, for samples doped with 2,4 , and $16 \mathrm{~g} / 1$ solution concentration. For the lightly doped samples, Fig. 7 shows that the electrical conductivity aging is such that it initially decreases with the aging time, reaches a minimum around the fifth day, and then slowly rises up to a value close to that of the undoped sample, namely, $\sigma=15.13 \times 10^{-12} \mathrm{mho} / \mathrm{cm}$, where it saturates. In other words, the main effect of the light iodine doping is to produce a decrease of the sample electrical conductivity which seems to be consistent with decrease of the average length of the polymer segments, as suggested by the analysis of the optical-absorption spectra aging. In contrast, for the heavily doped samples, the

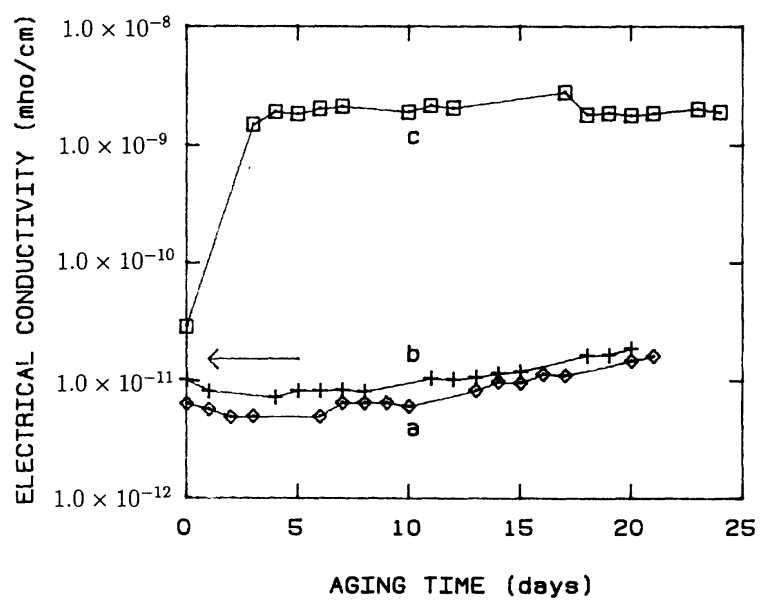

FIG. 7. Time evolution of the electrical conductivity, at 1 $\mathbf{k H z}$, of vulcanized cis-1,4-polyisoprene samples treated with iodine-doping solution concentrations of $2(a), 4(b)$, and $16(c)$ $\mathrm{g} / \mathrm{l}$. The arrow in this plot points at the undoped sample electrical conductivity. 
electrical conductivity aging is manifested by a monotonic rise to a saturation value which is reached after roughly one week aging time.

In Figs. 8 and 9 we summarize the results of our measurements on aged samples of the dielectric constant $\epsilon / \epsilon_{0}$, and the electrical conductivity $\sigma$ recorded at $1 \mathrm{kHz}$ as a function of the doping concentration. The data plotted in these figures incorporate the aging effects discussed above, in the sense that for samples doped with solution concentrations smaller than $6 \mathrm{~g} / 1$, the measurements were done on five-days aged samples. For concentrations greater than $6 \mathrm{~g} / \mathrm{l}$, the measurements were carried out on samples left aging for 10 days. Figure 8 shows that, up to a concentration of $6 \mathrm{~g} / \mathrm{l}$, the dielectric constant remains practically constant around the value of 2.35 corresponding to that of the undoped sample. For concentrations greater than $6 \mathrm{~g} / 1$, the dielectric constant begins to rise, upon doping, until it reaches a saturation value at high doping levels. The solid line in Fig. 8 represents the data fitting to a theoretical expression. The analytical expression which gave us the best data fitting was the logistic function of Eq. (1). The result found was

$$
\epsilon / \epsilon_{0}=1.95+17.24 \frac{\exp [(x-17.4) / 3.73]}{1+\exp [(x-17.4) / 3.73]} \text {. }
$$

The above results predict a saturation value for $\epsilon / \epsilon_{0}$ of 19.2 , implying that the iodine doping of vulcanized cis1,4-polyisoprene induces a drastic change in the dielectric constant of the native material. It changes from the low value of 2.35 , of the undoped material, to a value almost one order of magnitude larger, close to the values of the dielectric constants of some typical semiconductors, ${ }^{16}$ such as $\operatorname{InSb}(\epsilon=18)$ and $\mathrm{HgTe}(\epsilon=20)$, at high doping levels. Furthermore, the way this change takes place seems to be adequately described by a logistic function. This function belongs ${ }^{17}$ to a class of functions, the socalled $S$-shaped curves, describing rising functions approaching a saturation value with a change in their curvature. They are characterized essentially by two param-

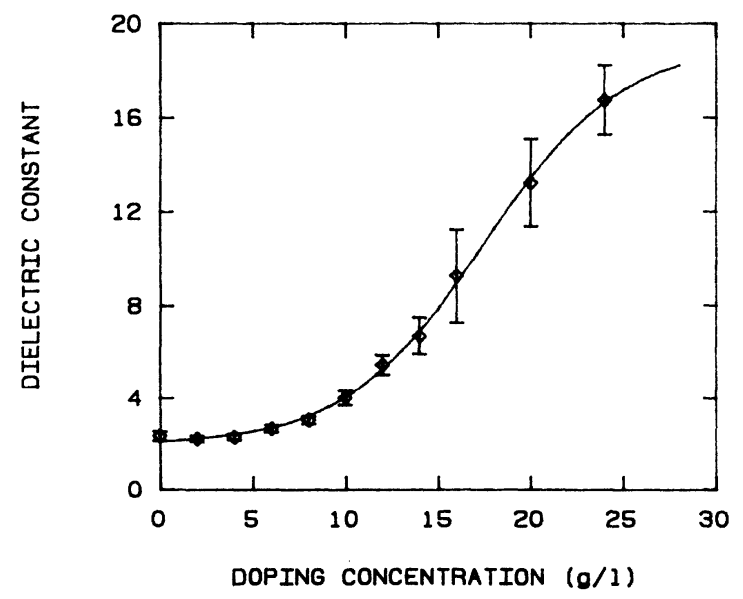

FIG. 8. Dielectric constant, recorded at $1 \mathrm{kHz}$, of iodinedoped vulcanized cis-1,4-polyisoprene samples, as a function of the doping solution concentration. The solid line represents the result of the data fitting to Eq. (2) of the text.

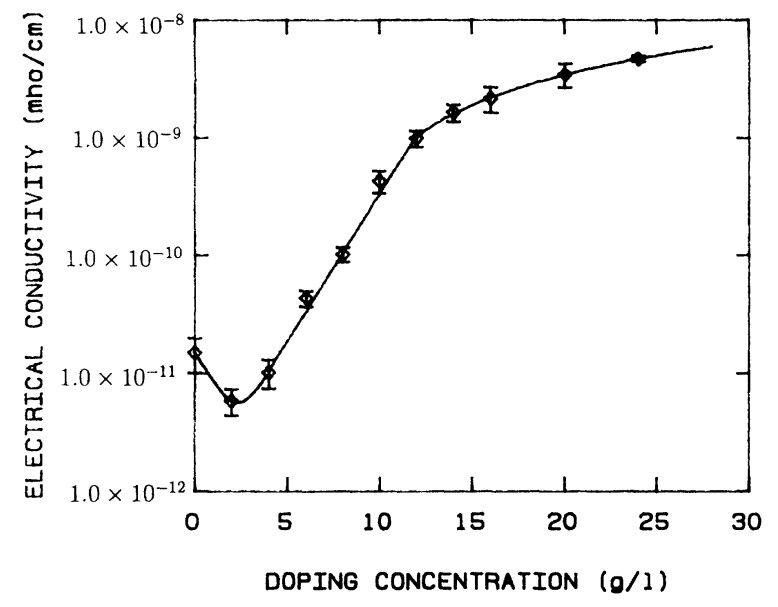

FIG. 9. Electrical conductivity, at $1 \mathrm{kHz}$, of iodine doped vulcanized cis-4,-polyisoprene samples, as a function of the doping solution concentration.

eters, namely, the point $x_{0}$, where the inflection occurs, and the characteristic length $\Delta x$ to approach saturation. They describe processes in which abrupt changes take place at a given position during a given interval, i.e., an "impulse" or "phase-transition"-like process. Formally the logistic curve [cf., Eq. (1)] is the solution of the Volterra-Lotka equation: ${ }^{18,19}$

$$
\frac{f^{\prime}}{f o}=\frac{1}{\Delta x} \frac{f}{f o}\left(1-\frac{f}{f o}\right),
$$

where $(\Delta x)^{-1}$ denotes the growth rate and fo is the saturation value, whose half-value is reached at $x_{0}$. Equation (3) means that the fractional rate of growth is proportional to the fraction $f / f o$, already grown times the fraction $(1-f / f o)$, left to grow, and it has been extensively used in market forecasting ${ }^{18}$ as well as in the study of ecological niches competition. ${ }^{19}$

A more marked dependence upon the doping concentration, was found in the electrical conductivity, as shown in Fig. 9. In the concentration range of $0-4 \mathrm{~g} / 1$, the electrical conductivity initially decreases, passes through a minimum at a concentration of $2.35 \mathrm{~g} / 1$, and then rises again; in this concentration range the dependence of $\sigma$ on the concentration was described by a second degree polynomion, represented, in Fig. 9, by the solid line. Between 4- and $12-\mathrm{g} / 1$ doping concentration, the electrical conductivity exhibits and exponential growth, which is followed by a saturation behavior, on further increasing the sample doping above the 12-g/1 concentration. The exponential growth and the saturation regime, described by a logistic function, were found from the data fitting in the corresponding concentration regions, and are represented in Fig. 9 by the solid lines. The initial decrease of the electrical conductivity at the light doping seems to be consistent with the hypothesis that the initial stage of the iodine doping is characterized by a decrease of the average length of the native polymer segments. If we assume a parabolic dependence of $\sigma$ on the doping concentration in this low-doping region, the electrical conductivity data, in units of $10^{-12} \mathrm{mho} / \mathrm{cm}$, 
are best fitted in the $0-4 \mathrm{~g} / 1$ by the following expression:

$$
\sigma=A-B x+C x^{2},
$$

where $A=15.13, B=8.03, C=1.71$, and $x$ denotes the doping concentration. Taking the derivative of Eq. (4) with respect to $x$, one finds

$$
\frac{d \sigma}{d x}=2 C x-B \text {. }
$$

According to this model, the rate of change of $\sigma$, in the low concentration region, results from the competition between gain and loss terms. The gain term is proportional to the iodine concentration and may be attributed to unbound iodine. The loss term in Eq. (4) reflects the presence of some trapping mechanism due probably to the segmentation of the native polymer matrix. The saturation of the electrical conductivity of our samples, predicted by the logistic function data fitting, occurs around $3.0 \times 10^{-8} \mathrm{mho} / \mathrm{cm}$. This value is roughly two thousand times higher than the undoped sample electrical conductivity. Even though the above factor indicates an appreciable change in $\sigma$, it is much smaller than those found in the previous works ${ }^{4,7}$ with nonvulcanized polyisoprene, namely, an increase by a factor of $10^{8}-10^{9}$. We attribute this relatively small change in the electrical conductivity of our samples to the fact that we have worked with a vulcanized material. In fact, our aim in this work was not to pursue the development of high performance conducting material, but rather, to investigate how the iodine doping affects the dielectric, the thermal and the mechanical properties of a more stable and chemically inert form of polyisoprene.

\section{THERMAL PROPERTIES}

The room-temperature thermal properties characterization of our $\mathbf{I}_{2}$-doped samples was based upon the measurements of the thermal diffusivity $\alpha$ and the thermal effusivity $e$. The thermal diffusivity, defined as $\alpha=k / \rho c$, measures essentially the thermalization time within the sample, whereas the thermal effusivity, $e=(k \rho c)^{1 / 2}$, measures the sample thermal impedance for the heat transfer. Here, $k$ denotes the sample thermal conductivity, $\rho$ is the materials density, and $c$ is the heat capacity at constant pressure. We note from these definitions, that the measurements of $\alpha$ and $e$ completely determine the sample's thermal properties, namely, $k$ and $\rho c$.

The importance of $\alpha$ as a physical parameter to be monitored is due to the fact that, like the opticalabsorption coefficient, it is unique for each material. This can be appreciated from the tabulated values of $\alpha$ presented by Touloukian et al. ${ }^{20}$ for a wide range of materials, such as, metal, minerals, foodstuffs, biological specimens and polymers. Furthermore, the thermal diffusivity is extremely dependent upon the effects of compositional and microstructural variables, ${ }^{21}$ as well as, processing conditions as in the case of polymers, ${ }^{22-24}$ glasses, ${ }^{25}$ metallic ribbons, ${ }^{26}$ semiconductors, ${ }^{27}$ and solvent evaporation. ${ }^{28}$

The thermal diffusivity can be accurately measured by the photoacoustic (PA) technique. ${ }^{11}$ The PA measure- ment of the thermal diffusivity of polymers has been reported by a number of authors. ${ }^{22-24,29,30}$ These papers employ different versions of the PA technique. Of the several PA techniques ${ }^{11}$ for measuring the thermal diffusivity we resorted to the "open cell" method described in Refs. 10 and 31 . It consists of mounting the sample directly onto a cylindrical electric microphone and using the front air chamber of the microphone itself as the usual gas chamber of conventional photoacoustics. Using the thermal diffusion model for the photoacoustic effect of Rosencwaig and Gersho ${ }^{11,32}$ in the present case of thermally thick samples, the thermal diffusivity $\alpha$, can then be obtained from the signal data fitting, in the high modulation frequency range, from the coefficient $a=\left(\pi l_{s}^{2} / \alpha_{s}\right)^{1 / 2}$ in the expression

$$
S=(A / f) \exp (-a / \sqrt{f}),
$$

where $l_{s}$ is the sample thickness.

The PA thermal diffusivity measurements were carried out using a 150-W halogen lamp whose polychromatic beam was modulated using a variable speed chopper (SRS model 540) and later focused onto the sample. The microphone output voltage was measured using a lock-in amplifier (SRS model 530). The thermal diffusivity values for the set of differents solutions concentrations doped samples were obtained from the signal data fitting of the Eq. (6) in the frequency range from 10 to $60 \mathrm{~Hz}$. In Fig. 10 we summarize the results for the thermal diffusivity of our iodine doped cis-1,4-polyisoprene samples, as a function of the doping concentration. As in the previous case of the dielectric measurements, the thermal diffusivity exhibits two distinct behaviors as a function of the iodine doping. At low doping levels, the thermal diffusivity decreases on increasing the doping concentration up to 6 $\mathrm{g} / \mathrm{l}$. At this point, the thermal diffusivity exhibits a sharp discontinuous growth towards a saturation at high doping concentrations. In fact, the thermal diffusivity data (in units of $10^{-4} \mathrm{~cm}^{2} / \mathrm{s}$ ) in the $0-6 \mathrm{~g} / 1$ concentration

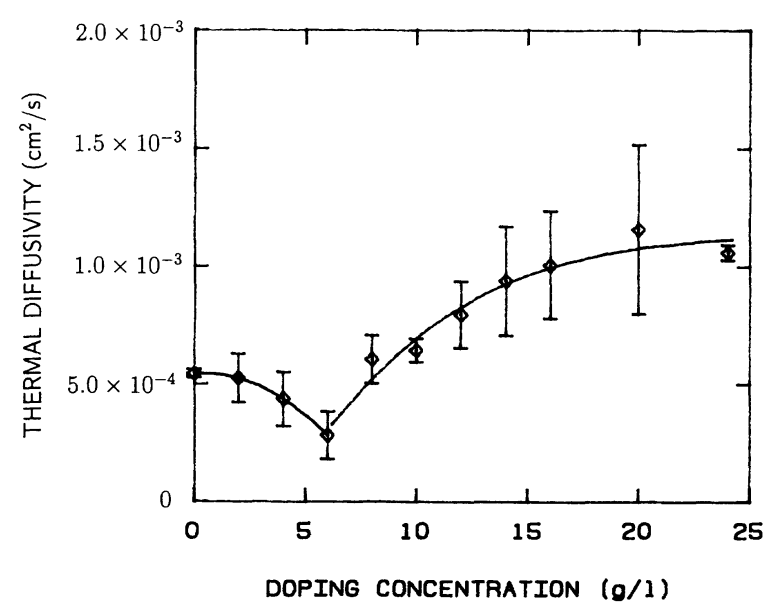

FIG. 10. Thermal diffusivity of iodine-doped vulcanized cis1,4-polyisoprene samples, as a function of the doping solution concentration. The solid lines represent the fitting of the experimental data to Eqs. (7) and (8) of the text. 
range, was best fitted, with an error of $0.05 \%$, by the following second degree polynomion:

$$
\alpha=5.44+0.073 x-0.085 x^{2},
$$

where $x$ denotes the iodine-doping concentration. This data fitting is represented in Fig. 10 by the solid line, in the corresponding concentration region. Above, the 6$\mathrm{g} / 1$ doping concentration, the thermal diffusivity (in units of $10^{-4} \mathrm{~cm}^{2} / \mathrm{s}$ ) was best fitted by a logistic function, namely,

$$
\alpha=-13.88+25.35 \frac{\exp [(x-2.608) / 4.843]}{1+\exp [(x-2.608) / 4.843]}
$$

represented, in Fig. 10, by the solid line for concentrations greater than $6 \mathrm{~g} / \mathrm{l}$.

It is well known ${ }^{20}$ that the thermal diffusivity of more organized and symmetric materials is greater than those of amorphous and less organized materials. The overall behavior of $\alpha$, shown in Fig. 10, may thus be thought as reflecting a discontinuous change in the materials symmetry. In other words, second-order phase transition takes place at the critical concentration of $6 \mathrm{~g} / 1$, as the doped material changes from a less organized state, at doping concentrations below $6 \mathrm{~g} / 1$, into a more organized state above this critical concentration. This suggestion that an order-disorder transition is taking place in our $\mathrm{I}_{2}$-doped cis-1,4,-polyisoprene samples is consistent with the hypothesis that, at the early stages, the iodine doping tends to break the native polymer segments, thereby making the material less organized and more flexible, as discussed in the previous sections. This tendency would manifest itself as a decrease of the thermal diffusivity, at low doping levels. In contrast, at high doping levels, the observed enhancement of the material's rigidity and electrical conductivity, indicate that some sort of structural organization takes place. This would imply an increase of the thermal diffusivity value, and is consistent with the generally accepted view ${ }^{33,34}$ that the final structure of conducting polymers consists essentially of a stack configuration of well-organized polymer segments with the dopant species forming regular arrays along the polymer chains.

To complete the sample thermal properties characterization, we have next measured the sample's thermal effusivity $e$. Knowing $e$ and $\alpha$, the sample thermal conductivity is readily obtained from the equation

$$
k=e \sqrt{\alpha} .
$$

The thermal effusivity was measured using the PA experimental setup described in Ref. 28. The pressure fluctuation in the PA cell can be calculated using the Rosencwaig and Gersho model for the photoacoustic effect, similarly to the previous case of the thermal diffusivity treated above. We refer to Ref. 28 for the details of the thermal effusivity measurements and calculations. The thermal effusivity may be straightforwardly obtained from the data fitting of the signal ratio $\delta P / \delta P_{0}$ as a function of the modulation frequency, where $\delta P_{0}$ is the pressure fluctuation caused just by the $A l$ absorber, and $\delta P$, the pressure fluctuation caused by the sample- $A l$ absorber system. Considering the relevant case of a thermally thin $A l$ absorber and a thermally thick sample, this signal amplitude ratio may be written as $^{28}$

$$
\left|\frac{\delta P}{\partial P_{0}}\right|=\frac{A}{\left[1+\left(b / z_{0}\right)+2\left(b / 2 z_{0}\right)^{2}\right]^{1 / 2}} .
$$

Here, $z_{0}=l_{0}\left(\pi f / \alpha_{0}\right)^{1 / 2}$, where $l_{0}$ is the thickness and $\alpha_{0}$ is the thermal diffusivity of the $A l$ absorber $\left(\alpha_{0}=0.92\right.$ $\left.\mathrm{cm}^{2} / \mathrm{s}\right) ; b=e / e_{0}$ is the ratio of the sample to the $A l$ foil thermal effusivity $\left(e_{0}=2.47 \mathrm{~W} \mathrm{~s}^{1 / 2} / \mathrm{cm}_{2} \mathrm{~K}\right)$; and the constant $A$ accounts for the eventual small differences in $A l$ foils reflectivities. $A$ and $b$ are left as fitting parameters.

The thermal effusivity values of the set of different solution concentrations doped samples were obtained from the signal amplitude ration data fitting to the Eq. (10) in the frequency range from 50 to $250 \mathrm{~Hz}$. From these values of $e$, using Eq. (9) and the previously measured value of $\alpha$, we have calculated the sample thermal conductivities. In Fig. 11 we summarize the results found for the thermal conductivity of our iodine-doped cis-1,4-polyisoprene samples, as a function of the doping concentration. Figure 11 shows that the thermal conductivity does not exhibit such a marked dependence upon doping as the thermal diffusivity and the electrical conductivity. In fact, the two main features of the $k$ dependence upon doping is a slight jump for the 6-g/1 doping concentration. Below this doping level, $k$ remain essentially constant at the value of $1.408 \pm 0.068 \mathrm{~mW} / \mathrm{cm} \mathrm{K}$; this is represented in Fig. 11 by the solid line in the corresponding doping region. For solution doping concentrations greater than $6 \mathrm{~g} / 1$, the thermal conductivity exhibits a tendency to decrease upon doping. In fact, the data in this concentration region, was best fitted by slowly decaying exponential function, namely,

$k=1.173+0.815 \exp (-x / 6.12) \quad($ in $\mathrm{mW} / \mathrm{cm} \mathrm{K})$.

The above results for the thermal conductivity, showing only a slight dependence upon the doping concentration, implies that the dramatic discontinuous change ob-

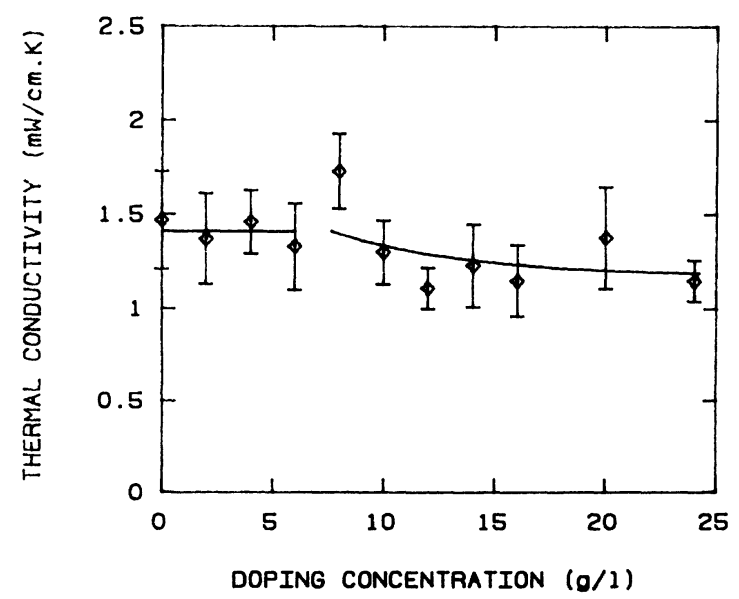

FIG. 11. Thermal conductivity of iodine-doped vulcanized cis-1,4-polyisoprene samples, as a function of the doping solution concentration. 


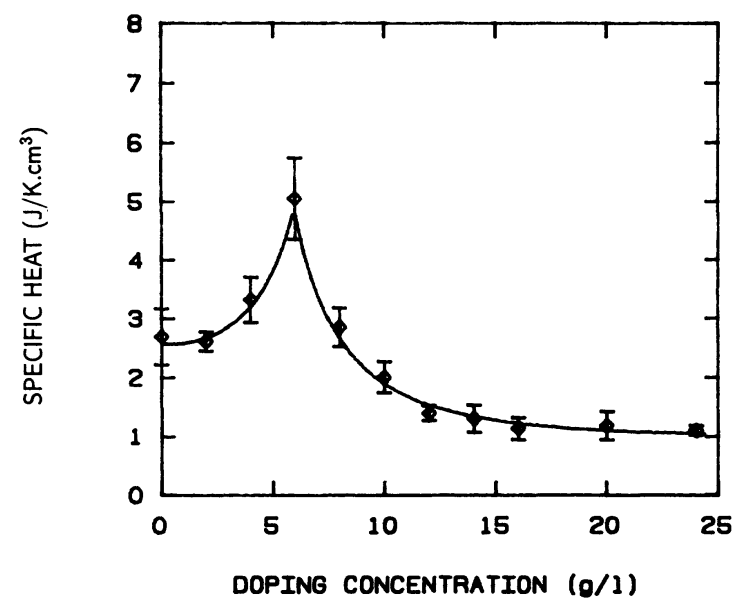

FIG. 12. Specific heat of iodine doped vulcanized cis-1-4,polyisoprene samples, as a function of the doping solution concentration.

served in the thermal diffusivity (cf., Fig. 10) around a 6$\mathrm{g} / 1$ concentration is essentially due to a jump in the sample specific heat, at this critical concentration. Using the data for $\alpha$ and $k$ given in Figs. 10 and 11, as well as the fact that $\rho c=k / \alpha$, we have calculated the specific heat of our samples for the several values of the doping solution concentration. The result we got is shown in Fig. 12, in which we also plot by solid lines the corresponding expressions for the specific heat, below and above the critical concentration, using the analytical expressions obtained from the $\alpha$ and $k$ data fitting, given by Eqs. (7), (8), and (11). Here, we note that for concentrations smaller than $6 \mathrm{~g} / \mathrm{l}$, we have used $k=1.408 \mathrm{~mW} / \mathrm{cm} \mathrm{K}$, in the specific-heat calculation.

The direction in which the specific heat changes discontinuously in Fig. 12 is from a larger value to a smaller value reflecting the way in which the materials symmetry changes, namely, from a less symmetric state to a state of higher symmetry. We note that the specific heat is smaller ${ }^{35}$ in a phase of higher symmetry. These results strongly support the foregoing suggestion that a second-order phase transition takes place in our vulcanized cis-1,4-polyisoprene samples, upon doping.

\section{TENSILE STRENGTH MEASUREMENTS}

Finally, as a further check on our findings and conclusions about the effects of the iodine incorporation into the vulcanized cis-1,4-polyisoprene samples, we have submitted our samples to tensile strength testing. The stress-elongation measurements were carried out on an Instron (model 1331) tensile testing machine at room temperature. The samples were cut in rectangular shape, typically $20 \times 10 \mathrm{~mm}^{2}$, and the load cells used corresponded to 50 and $500 \mathrm{~g}$, for the lightly and the heavily doped samples, respectively.

Using the statistical model ${ }^{36}$ for elastomers elasticity, the stress-elongation relation, at the small deformation regime, is given by

$$
\sigma_{t}=G\left[\lambda-\frac{1}{\lambda^{2}}\right],
$$

where the factor $G$ is given by $G=\rho R T / M_{c}$. Here, $\rho$ is the material's density, $T$ is the temperature, $R$ is the gas constant, and $\boldsymbol{M}_{c}$ is the average molecular mass of a chain length between crosslinks. For isotropic materials and constant volume deformations, the elastomer modulus $G$ is related to the Young's modulus $E$ by $E=3 G$. The expression for $G$ shows that it increases as the length between crosslinks is reduced ( $\boldsymbol{M}_{c}$ reduced). This means that the elastomer becomes stiffer as the crosslink density increases and the network becomes tighter.

The resulting values of $G$, obtained from the stresselongation curves for the several doped samples, are plotted in Fig. 13. This result provides us with further evidence for the conclusions drawn from the spectroscopic, electrical, and thermal properties measurements. Similarly to these properties, the elastomer modulus exhibited a drastic change on the doping concentration dependence at the critical 6-g/l concentration. Above this concentration, $G$ shows an exponential growth followed by a saturation plateau, as the doping concentration increases. The overall change in the modulus strength found was of roughly two orders of magnitude, corresponding to a change from $4.3 \times 10^{5} \mathrm{~Pa}$, for the undoped sample, to a value of $3.04 \times 10^{7} \mathrm{~Pa}$, for the heavily doped samples.

The exponential growth above the 6-g/ doping concentration means that the crosslink density is increasing upon doping, and, consequently, the material is getting more rigid. This agrees with the disappearance of the isolated $\mathbf{C}=\mathrm{C}$ bending and stretching modes of the native cis-1,4-polyisoprene, as shown in Fig. 4.

Furthermore, this crosslinking enhancement above the 6-g/l doping concentration, is also consistent with the observed increase in the electrical conductivity and thermal diffusivity, and the decrease of the specific heat; this may be viewed as reflecting the transition into a more ordered

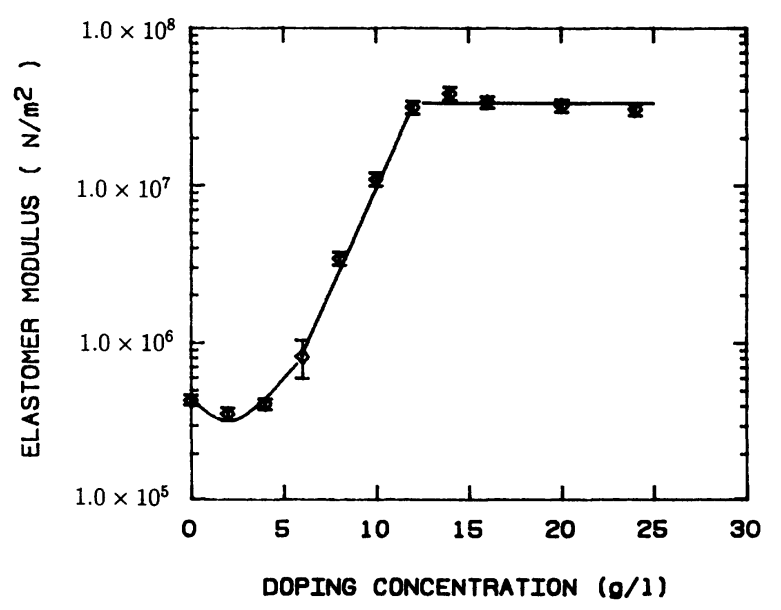

FIG. 13. Elastomer modulus of iodine doped vulcanized cis1,4-polyisoprene samples, as a function of the doping solution concentration. 
phase. Finally, at low doping levels, the decrease of the value of $G$, as compared to the undoped sample value, indicates a tendency towards the formation of a more amorphous phase. This confirms the observed shift towards short wavelengths and the decrease in the intensity of the optical-absorption spectrum, as shown in Fig. 1, as well as the initial decrease of the thermal diffusivity and electrical conductivity, shown in Figs. 10 and 9, respectively.

\section{CONCLUSIONS}

In this paper we have investigated the evolution of the dielectric and thermal properties of vulcanized cis-1,4polyisoprene samples upon doping. The changes of their physical properties were further supported by additional visible and infrared spectroscopy data as well as by tensile strength data. The previous works ${ }^{4-7}$ on iodine doping of polyisoprene were based upon (apart from the dc electrical conductivity) the information provided by optical and infrared spectroscopy data and magnetic resonance measurements. Here, apart from using the spectroscopy data, we report on the changes induced by iodine doping on the dielectric constant, thermal diffusivity, specific heat, and the elasticity modulus of vulcanized polyisoprene. These structure-sensitive physical parameters provide us information regarding the symmetry changes taking place in the material, supplying therefore important complementary information to the chemically sensitive optical and magnetic spectroscopy data.

As compared to the previous results, ${ }^{4-7}$ we have found a less dramatic, three orders of magnitude, increase in the electrical conductivity upon doping. The reasons for this are twofold. First, we have limited our sample doping to a relatively small level because of the eventual experimental difficulty in assembling the heavily doped samples in our dielectric and thermal properties measuring apparatus. The highly doped samples become rigid and somewhat corrugated. Secondly, our starting material and the doping process differed from the ones used in the previous works in the sense that we have worked with a more stable and chemically inert form of polyisoprene, namely, vulcanized natural rubber, doped in iodinemethanol solutions. Nevertheless, the overall results presented here seem to favor Dai and White's conclusion that iodine doping of polyisoprene produces, by doublebond shift reactions ${ }^{8,9}$ conjugated sequences of double bonds in the polyisoprene backbone, which are responsible for the observed changes in the electrical conductivity of these doped materials. This conclusion regarding the chemical transformations opposes those previously reported for which, due to radical cation ${ }^{4,5}$ and cationic complex ${ }^{6}$ formation, iodine-doped cis- $1,4,-$ polyisoprene is an example of nonconjugated conducting polymer. ${ }^{4-6}$

The additional information provided by the dielectric constant, thermal diffusivity, specific heat, and the elasticity modulus measurements reported above, indicate unequivocally the onset of an order-disorder transition in vulcanized polyisoprene, upon iodine doping. This was manifested by the sudden change in the behavior of these physical parameters for samples treated with $6-\mathrm{g} / 1$ doping solution concentration. Below this doping level, the electrical conductivity, the thermal diffusivity and the elastomer modulus all exhibit a decrease relative to the bare sample values, upon doping, whereas the specific heat increased. This behavior suggests that, at the early stage of iodine doping, the material is getting more amorphous in its structure which agrees with both the visually observed changes of its physical aspect (the samples doped with $2-\mathrm{g} / 1$ solutions are more flexible and sticky than the original undoped sample), as well as with the changes in the optical-absorption spectra. Above the critical doping concentration the optical-absorption spectra shift towards long wavelengths and increase their absorbance, at the same time that the vibrational bands of isolated $\mathrm{C}=\mathrm{C}$ bending and stretching modes decrease very rapidly, upon doping. These changes were followed by an increase of the electrical conductivity, thermal diffusivity, elasticity modulus, and a decrease in the specific heat, suggesting that the material moves into a more rigid, symmetric, and organized phase, due to an enhancement of interchain crosslinking. This orderdisorder transition in iodine-doped material agrees with the known structural changes that take place in other conducting polymers, ${ }^{33,34}$ in which their final structures consist essentially of a stack configuration of wellorganized polymer segments with the dopant species forming regular arrays along the polymer chains.

\section{ACKNOWLEDGMENT}

The authors are grateful to the Brazilian agency Conselho Nacional de Desenvolvimento Científico e Tecnológico for the partial financial support of this work.
${ }^{1}$ H. Shirakawa and S. Ikeda, Polym. J. 2, 231 (1971).

${ }^{2}$ H. Shirakawa, E. J. Louis, A. G. McDiarmid, C. R. Chiang, and A. J. Heeger, J. Chem. Soc. Chem. Commun. 578 (1977).

${ }^{3}$ N. Basescu, Z. X. Lin, D. Moses, A. J. Heeger, H. Naarmann, and N. Theophilou, Nature (London) 327, 403 (1987).

${ }^{4} \mathrm{M}$. Thakur, Macromolecules 21, 661 (1988).

${ }^{5}$ M. Thakur and B. S. Elman, J. Chem. Phys. 90, 2042 (1989).

${ }^{6}$ Q. Y. Shang, S. Pramanick, and B. Hudson, Macromolecules 23, 1886 (1990).

${ }^{7}$ L. Dai and J. W. White, Polymer 32, 2120 (1991).
${ }^{8}$ S. W. Benson and A. N. Bose, J. Am. Chem. Soc. 85, 1385 (1963).

${ }^{9}$ P. E. Sonnet, Tetrahedron 36, 557 (1980).

${ }^{10}$ L. F. Perondi and L. C. M. Miranda, J. Appl. Phys. 62, 2955 (1987).

${ }^{11}$ H. Vargas and L. C. M. Miranda, Phys. Rep. 161, 43 (1988).

${ }^{12}$ Y. Shindo and T. Hirai, Makromol. Chem. 155, 1 (1972).

${ }^{13}$ M. A. Rodrigues, M.S. thesis, University of Campinas, 1984 (unpublished).

${ }^{14}$ Y. Shindo, B. E. Read, and R. S. Stein, Makromol. Chem. 118, 
272 (1968).

${ }^{15}$ E. R. Blout, M. Fields, and R. Karplus, J. Am. Chem. Soc. 70, 194 (1948).

16J. I. Pankove, Optical Processes in Semiconductors (PrenticeHall, Englewood Cliffs, NJ, 1971).

${ }^{17}$ M. D. Silva and L. C. M. Miranda, Am. J. Phys. (to be published).

${ }^{18}$ J. C. Fisher and R. H. Pry, Technol. Forecast. Soc. Change 3, 75 (1971).

${ }^{19}$ I. Prigogine and I. Stengers, Dialog mit der Natur: Neue Wege Naturwissenschaftichen Denkes (Piper-Verlag, Munich, 1981).

${ }^{20}$ Y. S. Touloukian, R. W. Powell, Y. C. Ho, and M. C. Nicolasu, Thermal Diffusivity (IFI/Plenum, New York, 1973).

${ }^{21}$ G. Ziegler and D. P. H. Hasselman, J. Mater. Sci. 16, 495 (1981).

${ }^{22}$ B. Merté, P. Korpiun, E. Lüscher, and R. Tilgner, J. Phys. (Paris) Colloq. 44, C6-463 (1983).

${ }^{23}$ A. Torres-Filho, L. F. Perondi, and L. C. Miranda, J. Appl. Polym. Sci. 35, 103 (1988).

${ }^{24}$ N. F. Leite, A. H. Franzan, A. Torres-Filho, and L. C. M. Miranda, J. Appl. Polym. Sci. 39, 1361 (1990).
${ }^{25}$ A. C. Bento, H. Vargas, M. M. F. Aguiar, and L. C. M. Miranda, Phys. Chem. Glasses 28, 127 (1987).

${ }^{26}$ R. Kordecki, B. K. Bein, and J. Pelzl, Can. J. Phys. '64, 1204 (1986).

${ }^{27}$ A. Pinto Neto, H. Vargas, N. F. Leite, and L. C. M. Miranda, Phys. Rev. B 40, 3924 (1989).

${ }^{28}$ L. C. M. Miranda and N. Cella, Phys. Rev. B 47, 3896 (1993).

${ }^{29}$ A. H. Franzan, N. F. Leite, and L. C. M. Miranda, Appl. Phys. A 50, 431 (1990).

${ }^{30}$ A. Lachaine and P. Poulet, Appl. Phys. Lett. 45, 953 (1984).

${ }^{31}$ M. D. Silva, I. N. Bandeira, and L. C. M. Miranda, J. Phys. E 20, 1476 (1989).

${ }^{32}$ A. Rosencwaig and A. Gersho, J. Appl. Phys. 47, 64 (1976).

${ }^{33}$ G. Wegner, in Electronic Properties of Polymers and Related Compounds, edited by H. Kuzmany, M. Mehring, and S. Roth, Springer Series in Solid State Science Vol. 63 (Springer-Verlag, Berlin, 1985), p. 18.

${ }^{34}$ M. A. Druy, C. H. Tsang, N. Brown, A. J. Heeger, and A. G. McDiarmid, J. Polym. Sci.-Polym. Phys. Ed. 18, 429 (1980).

${ }^{35}$ L. D. Landau and E. M. Lifshitz, Statistical Physics (Pergamon, Oxford, 1970).

${ }^{36}$ P. C. Hiemenz, Polymer Chemistry (Dekker, New York, 1984). 